\title{
Vitamin B2 blocks development of Alzheimer's disease in APP/PS1 transgenic mice via anti-oxidative mechanism
}

\author{
Rong Zhao ${ }^{1}$, Huajun Wang ${ }^{1}$, Chen Qiao', Kai Zhao ${ }^{2 *}$ \\ ${ }^{1}$ Department of Pharmacy, Affiliated Hospital of Jiangsu University, Jiangsu Zhenjiang, 212000, ${ }^{2}$ School of Basic Medicine and \\ Clinical Pharmacy, China Pharmaceutical University, Jiangsu Nanjing, 211198, People's Republic of China
}

*For correspondence: Email: xt1356@163.com

\begin{abstract}
Purpose: To study the effect of vitamin B2 (VB2) on the development of Alzheimer's disease (AD). Methods: Memory and learning abilities were assessed in amyloid precursor protein/presenilin-1 (APP/PSI) transgenic mice aged 3 months, and C57BL/6 mice, using Morris water maze test. Brain tissue levels of key antioxidant enzymes, malondialdehyde (MDA), and reactive oxygen species (ROS) were assayed using ELISA kits. Western blot assay was used to monitor the expressions of Nrf2 and Keap1 proteins.

Results: Treatment with VB 2 significantly improved cognitive function of APP/PS1 mice with respect to number of crossings, duration of stay in target quadrant $(T Q)$, and escape latency time $(p<0.01)$. Moreover, VB2 also significantly reduced the levels of MDA and ROS. It also brought about significant increases in brain activities of CAT, SOD, and GSH-Px in APP/PS1 mice, relative to the control group ( $p$ $<0.01)$. Moreover, VB2 up-regulated Nrf2 expression but down-regulated the expression of Keap1 in brain tissue of transgenic mice.

Conclusion: These results indicate that VB2 protects the brain from ROS-induced AD damage, most likely due to its potential anti-oxidant property and activation of the Nrf2 pathway.
\end{abstract}

Keywords: Alzheimer's disease, Cognitive function, Vitamin B2, Reactive oxygen species, Superoxide oxide dismutase, Anti-oxidation

\begin{abstract}
This is an Open Access article that uses a funding model which does not charge readers or their institutions for access and distributed under the terms of the Creative Commons Attribution License (http://creativecommons.org/licenses/by/4.0) and the Budapest Open Access Initiative (http://www.budapestopenaccessinitiative.org/read), which permit unrestricted use, distribution, and reproduction in any medium, provided the original work is properly credited.

Tropical Journal of Pharmaceutical Research is indexed by Science Citation Index (SciSearch), Scopus, International Pharmaceutical Abstract, Chemical Abstracts, Embase, Index Copernicus, EBSCO, African Index Medicus, JournalSeek, Journal Citation Reports/Science Edition, Directory of Open Access Journals (DOAJ), African Journal Online, Bioline International, Open-J-Gate and Pharmacy Abstracts
\end{abstract}

\section{INTRODUCTION}

Alzheimer's disease (AD) is caused by degeneration of neurons, and manifests in patients in the form of progressively increasing memory loss, spatial disorientation, and dramatic slowdown in intellectual potency. These are the primary causes of dementia and disabilities (in old age) which impose enormous socioeconomic burdens on many countries with rapidly growing elderly populations [1]. Although the pathogenesis of this disease has not been fully elucidated, several competing hypotheses have been proposed. These include amyloidbeta $(A \beta)$ protein accumulation, cholinergic neurotransmitter deficiency, neurofibrillary tangles (NFTs) of tau proteins, increased inflammatory response, elevated reactive oxygen free radicals, and disordered ion dynamic equilibrium [2]. 
Increasing evidence indicate that oxidative stress is a key factor in the etiology of neurodegenerative diseases [3]. During the development of $A D$, oxidants are liberated in the brain, leading to excessive oxidative stress [4]. As products of aerobic metabolism, the levels ROS are controlled by the antioxidant and intracellular enzymes CAT, SOD and GSH-Px [57]. Under normal physiological conditions, ROS as signaling molecules are present at low levels in a transitory manner. However, excess accumulation of ROS leads to peroxidation, which does serious damage to DNA, phospholipids, and proteins. These damages are able to decrease the oxidase potency of mitochondrial cytochrome $\mathrm{C}$ and result in metabolic disturbance and cell apoptosis [8]. Thus, considerable research interest has been associated with the suppression of oxidative stress as a novel strategy for prevention and treatment of $A D[9,10]$. However, the current antioxidative drugs used for $A D$ patients have not achieved satisfactory efficacies in clinical trials. As a result, there is need for new anti-oxidative drugs for treating $A D$. At the present, a lot of attention has been devoted to natural bioactive products as likely candidates for treating $A D$ or ameliorating its symptoms $[11,12]$.

Vitamin B2 (VB2, Figure 1), also known as riboflavin, is widely distributed in animals and vegetables. It is of benefit to human health, through its diverse functions in biological oxidation, metabolism of vitamin B6, energy and cell growth. It is also associated with the absorption, storage and mobilization of iron in the body [13]. Moreover, VB2 has proven antioxidant activity $[14,15]$.

The present study was aimed at investigating the influence of VB2 on the development of $A D$ pathologies using an animal (mouse) AD model.<smiles>Cc1cc2nc3c(=O)[nH]c(=O)nc-3n(C[C@H](O)[C@H](O)[C@H](O)CO)c2cc1C</smiles>

Figure 1: Structure of vitamin B2

\section{EXPERIMENTAL}

\section{Animals and chemical reagents}

Male APP/PS1 double transgenic mice (32) aged 3 months old and weighing $26-28 \mathrm{~g}$ (Nanjing Biomedical Research Institute, Nanjing, China, license No. SYXK (Su) 2017009) were used as the experimental group. Eight (8) male C57BL/6 mice of the same age (Animal Center of Yangzhou University, China) were used as controls. The experimental procedures used in this investigation were approved by the Animal Experiment Committee of Jiangsu University of China (approval no. 2017-012). The experimental procedure followed the US NIH Guide for the Care and Use of Laboratory Animals [16].

A stock suspension $(3 \mathrm{mg} / \mathrm{ml}$ ) of VB2 (SigmaAldrich, USA) was prepared in $0.5 \%$ carboxymethyl cellulose sodium (CMC-Na), and administered via the intra-gastric route at a dose of $10 \mathrm{~mL} / \mathrm{kg}$ body weight. The activity of lactate dehydrogenase (LDH), catalase (CAT), GSH-Px, SOD, and levels of MDA were determined with appropriate assay kits provided by Jiancheng Institute of Bioengineering (Nanjing, China).

\section{Drug treatment}

The mice were randomly assigned to five groups (12 per group) and treated as indicated below:

Group I: CMC-Na (0.5 \%; $10 \mathrm{~mL} / \mathrm{kg})$ was administered to C57BL/6 male mice.

Group II: CMC-Na (0.5 \%; $10 \mathrm{~mL} / \mathrm{kg})$ was administered to APP/PS1 transgenic mice.

Group III: Memantine- $\mathrm{HCl}$ was dissolved in CMC$\mathrm{Na}(0.5 \%)$ and given to the APP/PSI double transgenic mice at a dose of $5 \mathrm{mg} / \mathrm{kg}$ body weight.

Groups IV and V: APP/PS1 mice received daily injections of VB2 at two doses (10 and 30 $\mathrm{mg} / \mathrm{kg}$ ). All treatments were given intra-gastrically daily for 70 days, and the animals were assessed for behavioral parameters as from day 64 .

\section{Morris water maze test}

Memory and learning were evaluated in the mice using the Morris water maze test, but with some modifications $[17,18]$. The water maze comprised a cylindrical tank brimming with water maintained at a temperature of $23 \pm 1^{\circ} \mathrm{C}$. It was partitioned equally into 4 quadrants labeled east, west, north and south and east, and a 10-mm diameter $2 \mathrm{~cm}$ 
beneath water within quadrant in the east. This was designated the target quadrant (TQ). Training was conducted for 5 days, at the rate of 4 trials per day, and the escape latency was recorded from day 64 to day 68 of the study. On day 68 , the number of crossings and the time spent in TQ were noted and recorded according to a previously defined procedure [19].

\section{Assay of CAT, SOD, and GSH-Px activities, and determination of ROS and MDA}

Mice were sacrificed by decapitation 60 min after the final behavioral tests. The brain samples were carefully excised and dried with filter paper. Nine volumes of cold saline were used for preparation of $10 \%$ brain homogenate which was subjected to centrifugation for $10 \mathrm{~min}$ at $2500 \mathrm{rpm}$. The supernatant was preserved at 4 ${ }^{\circ} \mathrm{C}$ for determination of ROS and MDA levels, as well as CAT, SOD, and GSH-Px activities using appropriate ELISA kits as per manufacturers' protocols.

\section{Western blot assay}

The procedure for Western blotting was based on the method reported previously [20]. Protein estimation in brain tissue of APP/PSI mice was done using BCA method. The proteins were separated on a gradient SDS-PAGE and then transferred to a PVDF membrane, and blocked for $1 \mathrm{~h}$ with $5 \%$ skim milk in TBST buffer (Tris$\mathrm{HCl}, 10 \mathrm{mM}, \mathrm{pH}$ 8.0, containing $0.1 \%$ Tween 20 and $150 \mathrm{mM} \mathrm{NaCl}$ ) (to block non-specific binding). This was followed by overnight incubation of the membrane with the primary antibody at $4{ }^{\circ} \mathrm{C}$. After this, the membrane underwent washing thrice with TBST buffer, followed by incubation for $2 \mathrm{~h}$ with secondary antibody at room temperature. After washing thrice, the protein bands were detected using enzyme-linked chemiluminescence kits according to the kit instructions. $\beta$-actin was used as the internal reference. The intensities of the resultant bands were quantified using Quantity One software. The expression of protein was depicted in terms of the ratio of the intensity of target protein band to the intensity produced by the $\beta$-actin band.

\section{Statistical analysis}

Data are expressed as mean \pm standard deviation (SD). They were analyzed with oneway analysis of variance (ANOVA), and Tukey's multiple comparisons. All analyses were carried out with GraphPad Prism 6.0. Values of $p<0.05$ were taken as indicative of statistically significant differences.

\section{RESULTS}

\section{Effect of VB2 on cognitive function of APP/PS1 mice}

Treatment with VB2 led to a significant reduction in escape latency time on day 68, when compared with day 64 in group I, indicating normal spatial learning capacity. However, the APP/PS1 mice with impaired learning had a significantly longer escape latency time on day 68, when compared with group I. Daily VB2 administration significantly and dose-dependently cut down the escape latency time of APP/PS1 mice on day 68 (Figure $2 \mathrm{~A}$ ).

Moreover, when compared with the other groups, the time spent by Group 1 mice in TQ was significantly longer, relative to the other quadrants on day 68, suggesting normal memory. In contrast, APP/PS1 mice displayed a significant decrease in time spent in TQ on day 68 and showed fewer crossings in the platform area than mice in group I (Figure $2 \mathrm{~B}-\mathrm{C}$ ). However, daily VB2 administration led to significant and dose-dependent improvement in the time spent in TQ on day 68 and the number of platform area crossings by APP/PS1 mice in groups III and IV, to extents comparable to the positive group III.

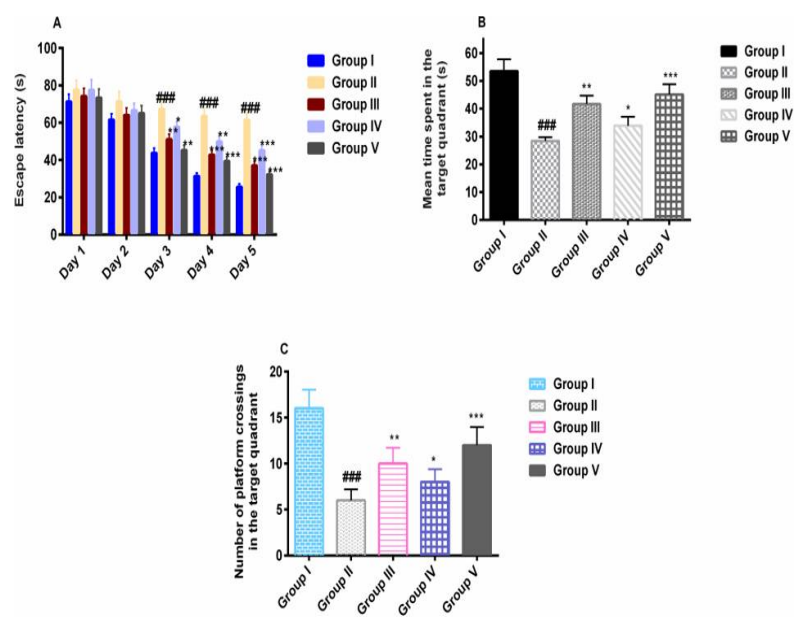

Figure 2: Effect of VB2 on memory and learning abilities of APP/PS1 mice. (A) Escape latency time during an acquisition trial in the Morris water maze. (B) Mean time spent in TQ on day 68. (C) Frequency platform crossings in TQ on day 68 during a retrieval trial in the Morris water maze. Values are mean \pm SD $(\mathrm{n}=12) ;{ }^{\# \#} p<0.001$, compared to group I; ${ }^{\star} p<0.05$, ${ }^{\star *} p<0.01,{ }^{\star * *} p<0.001$, relative to group II

\section{Effect of VB2 on ROS and MDA levels}

The level of ROS in group II was significantly increased, relative to the control group (Figure 3). However, the VB2-treated groups had 
significant and dose-dependent reduction in ROS levels, relative to group II. Besides, the ROS levels in groups IV and $\mathrm{V}$ were similar to that in the positive group (group III). A similar trend was seen in MDA levels.
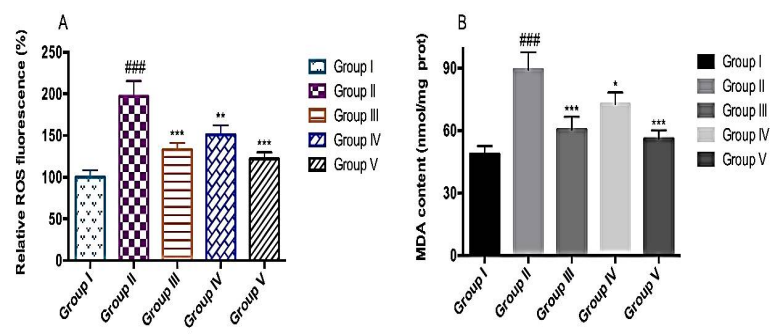

Figure 3: Effect of VB2 on ROS and MDA levels in brain tissues of APP/PS1 mice. (A) Relative ROS fluorescence; (B) MDA content. Values are mean \pm SD $(\mathrm{n}=12) ;{ }^{\# \#} p<0.001$, compared to group I; ${ }^{*} p<0.05$, ${ }^{\star *} p<0.01,{ }^{* \star *} p<0.001$, compared to group II

\section{Effect of VB2 on the activities of GSH-Px, CAT and SOD}

The results on Figures $4 \mathrm{~A}-4 \mathrm{C}$ show that in group 11 mice, there were significant decreases in GSH-Px, CAT and SOD levels, relative to their activities in group I. However, the activities of these antioxidant enzymes were significantly and dose-dependently increased by daily VB2 administration, when compared with corresponding activities in group II $(p<0.05)$ (Figures 4A - 4C).
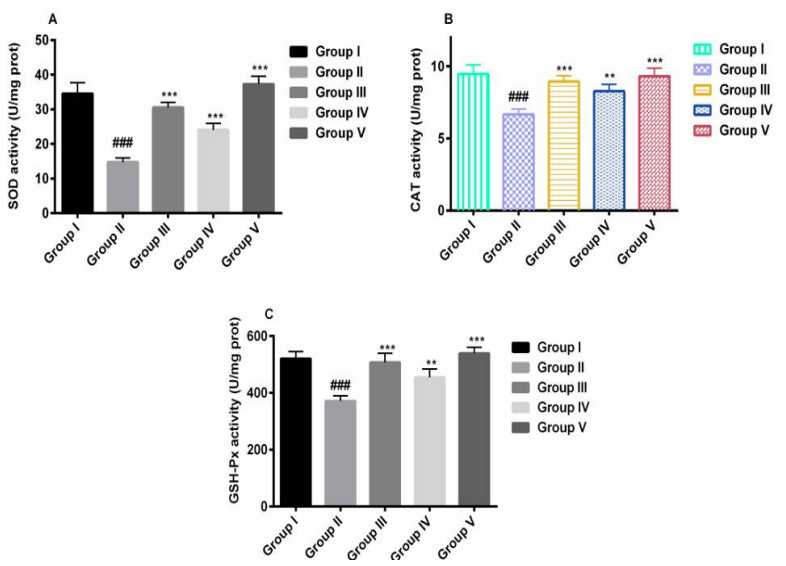

Figure 4: Effect of VB2 on the levels of SOD, CAT and GSH-Px in APP/PS1 mice, as assessed by ELISA. Values are mean $\pm S D(n=12) ;{ }^{\# \#} p<0.001$, compared to group I; ${ }^{*} p<0.05,{ }^{* *}<0.01,{ }^{* * *} p<0.001$, compared to group II

\section{Effect of VB2 on Nrf-2 and keap-1 expressions}

As indicated in Figure 5, Nrf2 expression in group II was significantly lower than in group I, while Keap1 showed the reverse result.
Compared with group II, the Nrf2 expression in low or high dose-treated mice increased significantly, while Keap1 expression decreased significantly.
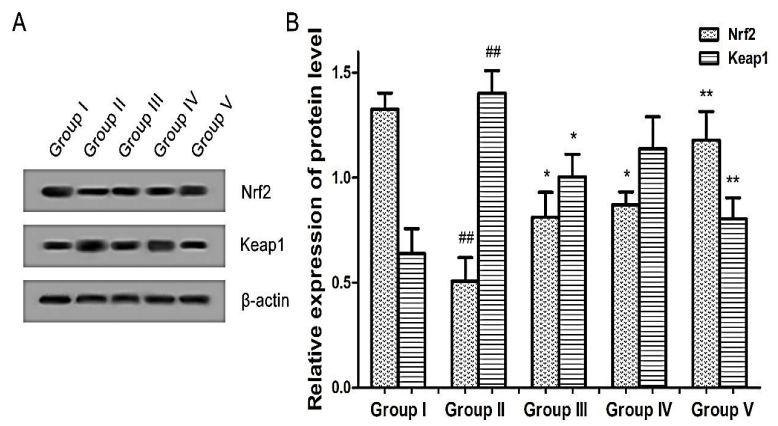

Figure 5: (A) Effect of VB2 on expression of Nrf2 and Keap1 in the brain tissues of APP/PS1 mice. (B) Relative expression of protein with respect to that of $\beta$ Tubulin. Values are mean \pm SD; ${ }^{\#} p<0.01$, relative to group I; * $p<0.05,{ }^{* *} p<0.01$, compared to group II

\section{DISCUSSION}

In this study, the experimental APP/PS1 mice had significantly damaged abilities of learning and memory characterized by increased levels of hippocampal amyloid beta, tau protein and oxidative stress, relative to age-matched C57BL/6 mice. These mice are ideal models for studying the mechanistic processes involved in AD [22,23]. Increasing attention has been focused on the beneficial effect of fighting oxidative stress on AD development, especially the effect of diverse natural nutrient products with memory- and cognition-promoting effects. Thus, the effect of VB2 on AD mice was investigated in this study. The results showed that daily VB2 administration at two different doses can significantly increase the number of crossings in the target platform, and reduce escape latency in APP/PS1 transgenic mice, suggesting that VB2 could ameliorate AD-induced impairment in spatial learning and memory. Increased production of free radicals and decreased endogenous antioxidants may accelerate membrane phospholipid breakdown, leading to lipid peroxidation and cellular dysfunction.

Excess ROS are considered as a common characteristic of $A D$. In addition, increased levels of MDA in APP/PS1 mice relative to wild-type C57 mice have been reported as indicators of higher levels of oxidative stress [24]. The major lipid peroxidation indicator, MDA can seriously damage the structure of cell membranes leading to cell damage in a manner similar to the effect produced by ROS. Therefore, the contents of ROS and MDA indirectly reflect the severity of free radicals. In this study, the ROS and MDA 
levels were markedly reduced by treatment of transgenic mice with VB2, indicating that VB2 ameliorated oxidative stress in the APP/PS1 mice.

The brain neurons are protected from ROSinduced oxidative damage by the antioxidant enzymes SOD, CAT and GSH-Px [25]. The results obtained in this study showed that SOD activity was significantly increased by VB2 administration in APP/PS1 mice, suggesting that VB2 exerts anti-oxidative stress effects through elevation of antioxidant enzyme activity.

Nrf2 is a nuclear transcription factor that regulates antioxidant activity in vivo, and is vital in the prevention and treatment of diseases of the cardiovascular, central nervous and digestive systems [26]. Under normal conditions, Nrf2 is combined with the inhibitory factor Keap 1 in the cytoplasm, which keeps it in the inactive state thereby preventing it from playing its signal transmission function [27]. However, under oxidative stress or oxidative phosphorylation in vivo, Nrf2 in the cytoplasm is activated, and then enters the nucleus and binds to related antioxidant response factor, activating Nrf2 pathway and facilitating the expression of downstream antioxidant genes for CAT, SOD, GSH-Px [28-30].

In this study, VB2 administration led to significant increases in Nrf2 expression and significant decreases in the expression of the inhibitory factor Keap1 in brain tissues of transgenic mice. Thus, VB2 promoted the expressions of downstream antioxidant genes and increased antioxidant activities, which suggests that the anti-AD effect of VB2 is dependent on activation of the Nrf2 pathway.

\section{CONCLUSION}

Administration of VB2 to APP/PS1 transgenic mice confers protection against ROS-induced damage and mitigates impaired cognitive and memory capacities in AD. This protective effect may be attributed to its potential anti-oxidant property and activation of the Nrf2 pathway. These findings indicate the potential for clinical application of VB2 in the management of Alzheimer's disease.

\section{DECLARATIONS}

\section{Conflict of Interest}

No conflict of interest associated with this work.

\section{Contribution of Authors}

We declare that this work was done by the authors named in this article and all liabilities pertaining to claims relating to the content of this article will be borne by the authors. Rong Zhao and Kai Zhao conceived and designed the study, Huajun Wang collected and analyzed the data, Chen Qiao wrote the manuscript. All authors read and approved the manuscript for publication.

\section{REFERENCES}

1. Reisa S, Elizabeth $M$, Keith J. The evolution of preclinical Alzheimer's disease: implications for prevention trials. Neuron 2014; 84: 608-622.

2. Shannon $L M$, David MH. Recent advances from the bench toward the bedside in Alzheimer's disease. Ebiomed 2015; 2: 94-95.

3. Elena $M$, Maria $C P$, Antonio $C$, Patrizia M. Oxidative stress in brain aging, neurodegenerative and vascular diseases: an overview. J Chromatogr B Analyt Technol Biomed Life Sci 2005; 827: 65-75.

4. Francesca M, Maria $C P$, Roberto M, Sara E, Cecilia $C$, Roberta C, Patrizia Me. Biomarkers of oxidative and nitrosative damage in Alzheimer's disease and mild cognitive impairment. Ageing Res Rev 2009; 8: 285.

5. William MS, Tatiana VB. Peroxidase-ROS interactions. Nonlinear Dyn 2015; 68: 413-430.

6. Klaus $A$, Heribert $H$. Reactive oxygen species: metabolism, oxidative stress, and signal transduction. Annu Rev Plant Biol 2004; 55: 373-399.

7. Malgorzata N, Andrzej G. The Role of the Reactive Oxygen Species and Oxidative Stress in the Pathomechanism of the Age-Related Ocular Diseases and Other Pathologies of the Anterior and Posterior Eye Segments in Adults. Oxid Med Cell Longev 2016; 2016: 3164734.

8. Golsa J, Niloufar A, Fariba K. Chitooligosaccharidemediated neuroprotection is associated with modulation of Hsps expression and reduction of MAPK phosphorylation. Int J Biol Macromol 2011; 48: 726-735.

9. José CST, Tiago BS, Paula A, Fernanda B. Alzheimer's disease and antioxidant therapy: how long how far? Curr Med Chem 2013; 20: 2939-2952.

10. Roberto R, Mark AS, Xinglong W, George P, Hyoung GL, Xiongwei Z, Robert BP. Molecular neuropathogenesis of Alzheimer's disease: an interaction model stressing the central role of oxidative stress. Future Neurol 2012; 7: 287-305.

11. Hao Z, Ping W, Yixue X, Libo L, Zhen L, Yunhui L. Allicin ameliorates cognitive impairment in APP/PS1 mice via Suppressing oxidative stress by Blocking JNK Signaling Pathways. Tissue Cell 2017; 50: 89-95.

12. Tatsunori O, Ran O, Mayumi T, Hidenobu S, Masayuki S, Manami I, Yukiko M, Taro Y, Kenjiro O, Yuji $K$. Cilostazol Suppresses A $\beta$-induced Neurotoxicity in $\mathrm{SH}$ - 
SY5Y Cells through Inhibition of Oxidative Stress and MAPK Signaling Pathway. Front Aging Neurosci 2017; 9: 337.

13. Hilary J. Riboflavin (vitamin B2) health. Am J Clin Nutr 2003; 77: 1352-1360.

14. Cristina M, Anna H, Juana F, Mariusz $P$, Concepcion V, Henryk $Z$. Effect of flour extraction rate and baking process on vitamin $B_{1}$ and $B_{2}$ contents and antioxidant activity of ginger-based products. Eur Food Res Technol 2009; 230: 119.

15. Kiyoka H, Harumi N, Kaori Y, Yasuji O. Antioxidant and prooxidant activities of $B$ group vitamins in lipid peroxidation. J Uoeh 2006; 28: 359-368.

16. Yvan T, Francesco P, Micheael HS, Ludger R. Ethical principles and standards for the conduct of human and animal biological rhythm research. Chronobiol Int 2004; 21: 161.

17. Richard M. Development of a water-maze procedure for studying spatial learning in the rat. J. Neurosci. Methods 11, 47-60. J Neurosci Methods 1984; 11: 47-60.

18. Lei L, Yushan L, Hui K, Liu L, Charles M, Ming X, Jiong $D$, Junying G, Gang $H$. Aquaporin-4 deficiency exacerbates brain oxidative damage and memory deficits induced by long-term ovarian hormone deprivation and D-galactose injection. Int $J$ Neuropsychopharmacol 2012; 15: 55-68.

19. Markus PK, Rafael S, Sakina S, Mathieu D, Robert W, Dean W, Stephanie S, Daisy A, Michael H. Pan-PPAR Modulation Effectively Protects APP/PS1 Mice from Amyloid Deposition and Cognitive Deficits. Mol Neurobiol 2015; 51: 661.

20. Mintao M, Mayu $H$. Myricetin attenuates lung inflammation and provides protection against lipopolysaccharide-induced acute lung injury by inhibition of NF-kB pathway in rats. Trop J Pharm Res 2017; 16: 2585-2593.

21. Truyen N, Paul N, Cecil BP. The Nrf2-Antioxidant Response Element Signaling Pathway and Its Activation by Oxidative Stress. J Biol Chem 2009; 284: 13291 13295.
22. Fabrizio $T$, Shumin L, Fortunato $B$, Sean $W$, Paul $M$, Ottavio A. Progressive age-related development of Alzheimer-like pathology in APP/PS1 mice. Ann Neurol 2004; 55: 801.

23. Miren $E$, Dmitry $P$, Ignacio $P$, Norma $A$, Teresa $C$, Carlos BZ, Mercè $P$, Carme A, Jaume F, Antoni C. Evaluation of Neuropathological Effects of a High-Fat Diet in a Presymptomatic Alzheimer's Disease Stage in APP/PS1 Mice. J Alzheimers Dis 2016; 54: 233-251.

24. Xiaoyan $T$, Chaonan J, Ying $L$, Yang $Y$, Shengnan $K$, Shaoshan $M$, Jie $M$, Junqing $Y$. PGE2-EP3 signaling pathway contributes to protective effects of misoprostol on cerebral injury in APP/PS1 mice. Oncotarget 2016; 7: 25304-25314.

25. Md MAA, Samiha A, Tasdid H, Tanzir A, SM NH, A RMS, Mohannad $S$. The antioxidant effect of astaxanthin is higher in young mice than aged: a region specific study on brain. Metab Brain Dis 2015; 30: 1237-1246.

26. Makoto K, Masayuki Y. Molecular mechanisms activating the Nrf2-Keap1 pathway of antioxidant gene regulation. Antioxid Redox Signal 2005; 7: 385.

27. Meng CL, Jian AJ, Zheng YJ, Qi DY. The Keap1-Nrf2ARE Pathway As a Potential Preventive and Therapeutic Target: An Update. Med Res Rev 2016; 36: 924-963.

28. Hozumi M, Masayuki Y. Nrf2-Keap1 defines a physiologically important stress response mechanism. Trends Mol Med 2004; 10: 549-557.

29. Sandra IM, Rui OC, Ildetd LF, Isabel S, Gladys C, Carmela $P$, Ana $C F$, Inês $B$, Cetarina $C$, Liliana $L$. Oxidative stress involving changes in Nrf2 and ER stress in early stages of Alzheimer's disease. Biochim Biophys Acta 2015; 1852: 1428-1441.

30. Fiona K, Oyinkan SA, Dobril KI, Jemma G, Beatriz GPN, Hélène $B$, Pedro $M$, Rebecca $C$, Inge $S$, Helena $M C$. Direct Keap1-Nrf2 disruption as a potential therapeutic target for Alzheimer's disease. Plos Genetics 2017; 13: e1006593. 